\title{
El Coaching como Herramienta de Trabajo de la Competencia Emocional en la Formación de Estudiantes de Educación Social y Trabajo Social de la Universidad Ramón Llull, España
}

\author{
Genoveva Rosa, Gisela Riberas, Lisette Navarro-Segura y Jesús Vilar \\ Universidad Ramón Llull, Facultad de Educación Social y Trabajo Social Pere Tarrés, Santaló 37, 08021 \\ Barcelona-España (e-mail: grosa@peretarres.org; griberas@peretarres.org; Inavarro@peretarres.org; \\ jvilar@peretarres.org)
}

Recibido Ene. 9, 2015; Aceptado Mar. 13, 2015; Versión final May. 15, 2015, Publicado Oct. 2015

\begin{abstract}
Resumen
Se presenta una propuesta de formación universitaria sobre la competencia emocional. La hipótesis de trabajo es que los profesionales de la acción social deben ser profesionales reflexivos ya que viven situaciones complejas que contienen componentes emocionales que añaden dificultad a las propias de aquella situación. Esto genera contradicciones y dilemas, por lo que la racionalidad técnica es necesaria, pero no suficiente. Esta experiencia se concreta a modo de ejemplo en la asignatura de Técnicas de Entrevista que se imparte en Educación Social y Trabajo Social de la Universidad Ramón Llull en España, donde se utilizan técnicas de coaching para trabajar la competencia emocional. A partir de cuestionarios y trabajos en grupo, se obtienen conclusiones que aportan datos sobre los resultados favorables del uso de metodologías integradas entre asignaturas, que contribuyen a la formación de profesionales reflexivos. Estas experiencias les capacitan para ejercer la profesión en entornos complejos de manera eficaz, responsable y proactiva ante situaciones potencialmente estresantes.
\end{abstract}

\section{Coaching as a Working Tool of Emotional Competence in the Formation of Students of Social Education and Social Work at the University Ramón Llull, Spain}

\begin{abstract}
This article presents a proposal for university training in emotional competence. The hypothesis of this work is that professionals in social action should be reflective practitioners, given that many of the complex situations with which they have to engage have emotional components which add to their inherent difficulties. In dealing with the contradictions and dilemmas that this generates, technical rationality is necessary but not sufficient. This training experience was carried out in the Interview Techniques section of the Social Education and Social Work majors, in which coaching techniques are utilized to work on emotional intelligence. The conclusions derived from questionnaires and focus group work provide data on the benefits of using integrated approaches among different subjects to contribute to the formation of reflective practitioners. These experiences enable practitioners to work in complex environments in an effective, responsible and proactive way under potentially stressful situations.
\end{abstract}




\section{INTRODUCCIÓN}

El profesional que se dedica a la acción social ha de estar capacitado para ser próximo a la persona que atiende y, a su vez, ha de saber protegerse para poder establecer una óptima relación de ayuda y prevenir el burnout ya que en muchos casos hablamos de situaciones multiproblemáticas. Para ello, como Viñas y Fabregó (2012) apuntan, es necesario iniciar en la formación de Educación Social y Trabajo Social un camino de autoconocimiento y de auto observación de las emociones para poder ser profesionalmente competentes, dada la complejidad de los casos sobre los que trabajan estos profesionales. Es necesario, por tanto, actuar desde la prevención de la propia salud del profesional así como desde la intervención más ajustada según las necesidades de los usuarios.

En este artículo se presenta una experiencia formativa de la Facultad de Educación Social y Trabajo Social Pere Tarrés de la Universidad Ramón Llull de Barcelona (España) donde se imparten los Grados de Educación Social y Trabajo Social. Esta experiencia se basa en el trabajo realizado con los estudiantes alrededor de la competencia emocional como una de las áreas fundamentales para el desarrollo integral de los profesionales vinculados a la intervención social y, concretamente, a la relación de ayuda, especialmente en situaciones críticas.

Esta experiencia forma parte de la línea estratégica de investigación: "Ética, responsabilidad social, competencias y técnicas en contextos complejos" que es una de las líneas de trabajo del Grupo Emergente de investigación de nuestra Facultad que lleva por nombre: Innovación y Análisis Social (GIAS). Esta línea está formada por 16 docentes de la Facultad. Tiene por objeto, en primer lugar, el desarrollo de propuestas de investigación para verificar hasta qué punto la formación que reciben los estudiantes a lo largo de los cuatro años es pertinente y suficiente en relación al tipo de competencias necesarias para desarrollar su actividad responsablemente y con eficacia en los entornos complejos donde trabajarán. En segundo lugar, esta línea pretende conocer las necesidades formativas y de asesoramiento de los profesionales en activo frente a este mismo tipo de situaciones. Los datos obtenidos en las distintas investigaciones llevadas a cabo hasta el momento, han permitido reorientar los planes de estudio de grado y desarrollar nuevas propuestas de formación permanente en forma de máster o postgrado, así como estructuras de soporte para los profesionales en activo, como pueden ser investigaciones compartidas o espacios de supervisión y de asesoramiento (Riberas et al., 2014).

En nuestra facultad formamos trabajadores sociales desde hace más de 80 años y educadores sociales desde que se creó esta figura profesional, en 1991. Nos dedicamos exclusivamente a la formación de estos dos Grados, por lo que somos expertos en lo que denominamos acción social. Bajo esta extensa trayectoria, nos hemos preocupado principalmente por ofrecer una formación de calidad vinculada a la realidad sobre la que los futuros profesionales se encontrarán una vez obtengan la titulación. Es por ello que cuidamos el acompañamiento que el alumno tiene a lo largo de los cuatro años que duran sus estudios a través de tutores que guían, acompañan y supervisan su recorrido.

Actualmente la Facultad ha obtenido la acreditación por la auditoría externa que realiza la Agencia de Calidad de Cataluña (AQU) y ocupa el primer puesto del estado español en el ranquin que realiza el diario El Mundo sobre las 50 carreras más demandadas del 2014-2015 y también ocupa esta posición en el Ranquin ISSUE de Indicadores Sintéticos del Sistema Universitario Español del 2015.

Tanto la profesión de Educación Social como la de Trabajo Social son profesiones con vocación de servicio que se desarrollan mayoritariamente en contextos de injusticia social y vulnerabilidad. Por ello requieren un nivel alto de vocación, un gran autoconocimiento, así como de gestión emocional y de situaciones complejas para gestionar situaciones como los conflictos de valor, muy frecuentes en su práctica profesional. De aquí que cada vez más se evidencie la importancia del trabajo alrededor de la competencia emocional como uno de los principales factores de éxito ante los retos de una sociedad compleja y ante la intervención con personas en riesgo de exclusión social. Se busca crear un profesional implicado, eficaz, eficiente, competente y que sepa sacar el máximo partido de los usuarios, normalmente en situaciones vulnerables, para que desde el empoderamiento pueda, a través de una relación de ayuda, acompañarlos hacia un mayor grado de autonomía y a su inserción en la sociedad. Partimos, en palabras de Lima (2012) de la necesidad de recrear nuevos recursos en la intervención social como los bienes relacionales, el empoderamiento, la participación... y repensar con criterios profesionales la calidad y ética de la intervención social teniendo como eje para ello a la persona.

Por todo ello, es imprescindible capacitar al futuro profesional con criterios que le ayuden a actuar ante situaciones éticamente complicadas, sobretodo en una época como la actual de gran diversidad, heterogeneidad y cambios constantes. El profesional necesita elementos que le permitan orientarse en este escenario complejo, bajo un prisma de justicia y con amplitud de miras, más allá de sus propias creencias. 
Desde este punto de vista el trabajo que se realiza en la Facultad parte de contar con un alto porcentaje de profesorado que combina la práctica profesional con la docencia, de manera que la formación sea cercana a la profesión desde la propia experiencia del profesorado. En este trabajo se da respuesta a esta necesidad y se aborda cómo la inteligencia emocional se puede plantear como una competencia transversal en las asignaturas, de manera que se realice un trabajo con los estudiantes que les capacite personal y profesionalmente para el establecimiento de climas positivos y creativos ante situaciones complejas. A su vez, se muestra cómo las herramientas del coaching ofrecen una metodología activa donde el rol del alumno es participativo, reflexivo y agente de cambio: perfil que por otro lado es el que requiere la sociedad en un escenario cambiante.

Debido a la transformación de la figura del profesional de la acción social como un agente reflexivo-creador (Riberas y Vilar, 2014) y teniendo en cuenta la evolución de la profesión de una perspectiva actuadora hacia una nueva perspectiva crítica y reflexiva, es necesario crear escenarios donde se prepare a los futuros profesionales a asumir los nuevos retos que emergen en una sociedad cambiante. La concepción actuadora del profesional tecnócrata se basa en el dominio de las habilidades y competencias tecnológicas, que permiten aplicar respuestas específicas de carácter resolutivo para cada uno de los problemas que pueden aparecer en los distintos escenarios de intervención dentro de una estructura de trabajo parcializada, donde la suma de actuaciones "correctas" asegura el éxito en la intervención. En contraposición, recuperando a Lima (2012) la formación del profesional reflexivo consiste en aportar los conocimientos que se aplicarán en cada una de las actuaciones específicas, teniendo en cuenta la dimensión global de la persona.

El planteamiento reflexivo-creador, por tanto, parte de una mirada global y compleja de la realidad. Se trata de una concepción donde los fenómenos se entienden como el resultado de las interacciones que se dan entre los diferentes elementos que la configuran. Ya no se trata de una mirada causal y lineal sobre la realidad, sino de llegar a entender que la interacción de los elementos que entran en contacto en cada escenario da como resultado una nueva realidad compleja con propiedades singulares, distintas y no reductibles a los elementos que la constituyen. Desde este punto de vista, la formación consiste en capacitar al profesional para crear conocimiento en un entorno complejo. Si comparamos los dos modelos, podemos constatar el cambio de paradigma y la necesidad de formación que emerge:

Tabla 1: Comparativa del profesional actuador versus el profesional reflexivo (Riberas et al., 2013; 32)

\begin{tabular}{|l|l|}
\hline Profesional actuador & Profesional reflexivo \\
\hline Lógica mecanicista & Lógica sistémica \\
Estructura tecnocrática y jerárquica & Estructura reflexiva y en red \\
Actuación finalista, resolutiva & Actuación dinamizadora, creativa \\
Aplicación mecánica de fórmulas prefijadas & La investigación como forma de crear conocimiento \\
Actividad reproductora & Actividad creadora \\
Trabajo solitario, "de corta mirada" & Trabajo cooperativo de "mirada amplia" \\
Trabajo organizado a partir de la lógica de los & $\begin{array}{l}\text { Trabajo organizado a partir de la necesidad de la } \\
\text { servicios }\end{array}$ \\
Multidisciplinariedad & persona atendida \\
\hline
\end{tabular}

Las principales manifestaciones del cambio del profesional actuador al reflexivo, se concretan en los siguientes puntos (Riberas y Vilar, 2014: 134): i) se produce el paso de una mirada centrada en la intervención resolutiva, parcial, a una mirada centrada en la promoción integral de la persona; ii) se entiende que un entorno complejo y a menudo difuso es el espacio natural de trabajo del profesional, para el cual no hay respuestas preestablecidas y es imprescindible crear respuestas "ad hoc" de cada situación; iii) para poder crear conocimiento, se pasa de la oposición teoría-práctica a una integración entre la reflexión y la acción; iv) para asegurar una correcta atención a las personas, es imprescindible un trabajo cooperativo de carácter interdisciplinario entre profesionales que evite caer en contradicciones entre los profesionales que intervienen en una misma realidad social; v) hay que asegurar el respeto a los procesos complejos y los tiempos de las personas atendidas, pero a la vez, hacer un uso adecuado de los recursos disponibles de manera eficaz y eficiente, y vi) se comprende que la actividad profesional es un ejercicio de responsabilidad moral que responde al compromiso adquirido con la sociedad de la que se forma parte.

En resumen, tal como muestra la producción académica desde los años 90 , los modelos clásicos de carácter tecnocrático que pretendían formar profesionales actuadores y resolutivos a partir de la fragmentación de la realidad en parcelas cerradas de actuación, se han manifestado insuficientes en la realidad actual (Gimeno, 1987; Sáez, 1997; Caride, 2003; Úcar, 2006). Hoy en día ya no se discute la necesidad de plantear la formación desde una perspectiva transversal que capacite a los profesionales para 
ser críticos, reflexivos e investigadores, si queremos que sean capaces de trabajar en escenarios de complejidad (Schön, 1998; Elliot, 1989; Stenhouse, 1987).Pero esta formación no es sencilla. De hecho, y siguiendo a Morin, "la dificultad del pensamiento complejo es que debe afrontar lo entramado (el juego infinito de retroalimentaciones), la solidaridad de los fenómenos entre sí, la bruma, la incertidumbre, la contradicción" (2008: 33). Hay que capacitar a los futuros profesionales para trabajar desde la creatividad y la investigación en su práctica (Imbernon, 2007; Perrenoud, 2004).

Los cambios sugeridos por el EEES (Espacio Europeo de Educación Superior), a pesar de las muchas contradicciones y aspectos criticables, han abierto una oportunidad de plantear cambios metodológicos que contribuyan a hacer una formación capacitadora para trabajar en escenarios de complejidad: "autoregulación, metacognición, relación de nuevos conocimientos con conocimientos previos, formulación y análisis de hipótesis, planteamiento de alternativas, evaluación de resultados..." (Trillo, 2008: 25). En nuestro caso, ha comportado una reorientación de la metodología formativa hacia un sistema basado en el aprendizaje integrado y transversal que revalorizan los aspectos prácticos y aplicados, en la línea que propone Salvador et al. (2011: 48) quien apuesta por una metodología activa en la formación universitaria dado que el alumnado "demanda claramente que se incorpore la vertiente práctica o aplicada en el desarrollo de las clases, en concreto mediante la realización de ejercicios individuales o grupales y estudio de casos".

\section{LA INTELIGENCIA EMOCIONAL}

La complejidad de las situaciones que afrontan y ayudan a afrontar los profesionales de la acción social requiere poner en juego conocimientos, procedimientos, actitudes y actuaciones estrechamente vinculadas a lo que Gardner (1987) denomina las inteligencias intrapersonal e interpersonal. Estas dos dimensiones de la inteligencia configuran el concepto de inteligencia emocional, ampliamente estudiado en las últimas décadas y base de numerosas publicaciones sobre el papel de la inteligencia emocional en el ámbito educativo. La mayoría de los estudios mencionan las teorías de Mayer y Salovey (1997), quienes definen la inteligencia emocional partiendo de sus cuatro componentes (percepción, asimilación, comprensión y regulación) como: "la habilidad para percibir, asimilar, comprender y regular las propias emociones y la de los demás promoviendo un crecimiento emocional e intelectual" (Mayer y Salovey, 1997: 10). Para Goleman (1995) la inteligencia emocional es más importante que el coeficiente intelectual y las habilidades técnicas para poderse desenvolver de manera efectiva en cualquier trabajo. La inteligencia emocional permite el desarrollo de la conciencia y el autoconocimiento de manera que se pueden gestionar las emociones, a la vez que permite un desarrollo óptimo de las habilidades sociales.

Concretamente autores como Extremera y Fernández-Berrocal (2004), sostienen que las personas emocionalmente inteligentes no sólo serán más hábiles para percibir, comprender y manejar sus propias emociones, sino también serán capaces de extrapolar sus habilidades de percepción, comprensión y manejo de las emociones de los demás, por lo que según los mismos, la inteligencia emocional jugaría un papel elemental en el establecimiento, mantenimiento y la calidad de las relaciones interpersonales. A su vez un elevado nivel de inteligencia emocional conlleva a un mejor control del estrés y a un aumento en el rendimiento académico.

Siguiendo estos mismos autores, existe una clara influencia entre las habilidades emocionales y estrategias de afrontamiento, como la supresión de pensamientos en la aparición del burnout y el desajuste emocional en el profesorado. Ello nos lleva a plantear que la propuesta formativa que se desarrolla pueda servir a su vez a la propia acción del profesorado, puesto que pone en juego sus propias habilidades intrapersonales e interpersonales en la acción formativa si pretende trabajar desde la inteligencia emocional sus asignaturas. Se establece pues un doble mecanismo: de adquisición de conocimientos y de autoconocimiento por parte del alumno y de prevención de burnout como futuro profesional con la adquisición de las herramientas necesarias.

Siguiendo a Tobón (2005), las competencias transversales, tal y como se recoge en los Libros Blancos de las titulaciones, se clasifican en: instrumentales, interpersonales y sistémicas. Como indican Rosa et al. (2012), además de las competencias intrapersonales e interpersonales, se destacan las competencias generales o sistémicas que se impulsan en la titulación como elementos esenciales para consolidar esta nueva posición profesional: capacidad de desarrollar la identidad profesional, capacidad ética y moral y capacidad de evaluación continua.

Estas competencias son las que permiten el traslado de una materia a otra. Concretamente se hace referencia a lo que autores como Bisquerra denominan competencia emocional, definida por el mismo como "factor esencial para la prevención y el desarrollo personal y social" (2003: 8). Para el autor, la competencia emocional se puede entender como el "conjunto de conocimientos, capacidades, habilidades y actitudes 
necesarias para comprender, expresar y regular de forma apropiada los fenómenos emocionales" (2003: 22). Dentro de la competencia emocional se pueden encontrar las competencias de autorreflexión o inteligencia intrapersonal y las capacidades de reconocer lo que los demás piensan y sienten, dicho de otra manera: la inteligencia interpersonal. Éstas, a su vez, se pueden agrupar en cinco bloques: conciencia emocional, regulación emocional, autonomía personal, inteligencia interpersonal y habilidades de vida y bienestar (Bisquerra y Pérez, 2007).

Como se ha comentado con anterioridad, la convergencia hacia el Espacio Europeo de la Enseñanza Superior (EEES) conlleva una reorientación de la metodología hacia un sistema basado en el aprendizaje y en la revalorización de los elementos prácticos y aplicados. Garantizar el alcance de las competencias profesionales, no se suscribe únicamente mediante la adquisición de conocimientos, procedimientos y actitudes, de manera que el proceso de formación ha de incluir necesariamente el desarrollo personal del alumnado. Hablamos pues de una nueva definición de las metodologías docentes. El nuevo modelo se centra en el aprendizaje del alumno más que en la enseñanza del profesor.

A partir de plantear una actividad formativa centrada en el estudiante, donde la adquisición de competencias será uno de los retos principales de cada propuesta formativa, se genera la necesidad de disponer de instrumentos metodológicos que rompan con el paradigma del rol tradicional del profesor como instructor. Por otro lado, y siguiendo a Delors (1996) y sus ya conocidos cuatro pilares de la educación del siglo XXI, (esto es, aprender a conocer, aprender a hacer, aprender a convivir y aprender a ser), vemos que estos dos últimos son los que tienen una relación directa con la educación emocional que planteamos en este artículo. Como apunta Bisquerra (2003) conocer las propias emociones, la relación que guardan éstas con nuestros pensamientos y comportamientos, debería ser uno de los objetivos de la educación, además de la necesaria incidencia sobre las relaciones interpersonales.

De este modo, el desarrollo de las competencias responde a una propuesta centrada en el alumno y en su capacidad de aprender; por ello se le exige más implicación y compromiso en un rol activo donde el profesor además de ser un experto en su disciplina, como señala Madrid (2005: 60), deberá contar con una amplia gama de competencias profesionales básicas: el conocimiento del proceso de aprendizaje del estudiante en contextos académicos, no formales e informales; la planificación de la enseñanza y de la interacción didáctica; la utilización de métodos y técnicas didácticas adecuadas; la gestión de interacción didáctica y de las relaciones con los estudiantes; la evaluación, el control y la regulación de la docencia y del aprendizaje; el conocimiento de normas legales sobre derechos y deberes del profesor y del estudiante, y gestión del desarrollo profesional docente.

La tipología de actividades se apoya en la atención a la diversidad, ya que cada alumno aprende de una determinada manera. Cabe tener presente esta variedad en las propuestas respetando los diferentes estilos de aprendizaje. Con nuestro despliegue de las asignaturas de los estudios de Educación Social y de Trabajo Social, (y como en el 2006 sugería la Secretaria de Estado de Universidades e Investigación) hemos impulsado una diversidad de actividades formativas que se recogen en las memorias verificadas de las titulaciones, dentro de lo que se puede definir como Aprendizaje Basado en Problemas. Tal como indican Fernández y Duarte (2013: 31) este tipo de planteamientos lleva a los estudiantes al aprendizaje de los contenidos de información de manera similar a la que utilizarán en situaciones futuras, fomentando que lo aprendido se comprenda y no sólo se memorice.

El Aprendizaje Basado en Problemas posibilita crear una variedad de actividades en las aulas como son: sesiones magistrales, exposiciones teóricas, estudio de casos, realización de simulaciones, análisis de documentación, desarrollo de tareas grupales, evaluación entre iguales, presentación de esquemas conceptuales y material específico, realización de debates, utilización de plataforma virtual, seguimiento personalizado del estudiante, vinculación con la praxis profesional y supervisión grupal entre otras. Esta variedad de actividades permite que cada docente adapte su metodología al tipo de asignatura y a su propio estilo de enseñanza.

Que en las diferentes asignaturas se realicen actividades similares o se realicen trabajos para más de una asignatura permiten consolidar conocimientos, desarrollar competencias y, a la vez, optimizar el esfuerzo de los estudiantes, al poder utilizar un trabajo como eje central que se adapta a las particularidades de las mismas. Se da, según Salvador, et al. (2011) una relación individualizada y diversa entre los procesos de enseñanza y los de aprendizaje.

Tal como indican Osuna y Luna (2008: 30) siguiendo a Hirsch (2005), los rasgos que caracterizan a las profesiones se pueden agrupar en cuatro grandes competencias: a) cognitivas y técnicas: aglutina rasgos concernientes a la adquisición de conocimientos tales como: la formación, preparación y competencia técnica; formación continua; innovación y superación; b) sociales: conformada por rasgos como 
compañerismo y relaciones; comunicación; trabajar en equipo y, ser trabajador; c) éticas: integrada por rasgos como responsabilidad; honestidad; ética profesional y personal; prestar mejor servicio a la sociedad; respeto, principios morales y valores profesionales. Y por último, d) afectivo emocionales: representadas por rasgos tales como identificación con la profesión y capacidad emocional.

\section{EL COACHING APLICADO A LOS ESTUDIOS DE EDUCACIÓN SOCIAL Y TRABAJO SOCIAL}

El perfil profesional de los egresados de las titulaciones de Trabajo Social y Educación Social requiere que se minimicen los riesgos emocionales que pueden tener en el ejercicio de la profesión y se preparen para afrontarlos. En toda relación de ayuda, la inteligencia emocional tiene un papel importante, por lo que incidir desde esta dimensión en la formación universitaria prepara al futuro profesional para trabajar con un amplio abanico de recursos que le servirán en la mejora de sus intervenciones enriqueciendo, a su vez, sus relaciones y su competencia comunicativa, respetando los objetivos con los que trabaja y reforzando la autoestima del usuario a la vez que la suya.

Desde la Universidad se plantea hacer frente a esta necesidad utilizando diversas técnicas y disciplinas que garanticen la consecución de las competencias relacionadas con esta dimensión. Estas competencias están recogidas en la memoria de ambas titulaciones y se concretan en: i) desarrollar habilidades cognitivas, estrategias de gestión de las emociones y habilidades de comunicación que le permiten afrontar adecuadamente las tensiones y conflictos interpersonales propios de la acción social y la relación de ayuda, ii) conocer los modos de intervenir con personas y grupos para potenciar la toma de decisiones y promover la participación de los usuarios en los procesos socioeducativos, iii) manifestar capacidad de contención emocional en momentos de tensión, en el ejercicio de la práctica profesional.

La experiencia de innovación metodológica sobre el coaching como estrategia formativa, los resultados obtenidos y el nivel de satisfacción de los estudiantes, que se muestran más adelante, nos hace creer que el modelo que aquí se presenta ha de ser planteado de manera transversal en las dos titulaciones. La finalidad es poder establecer una línea de trabajo que incida desde las diferentes asignaturas de los Grados en una formación integral de nuestros estudiantes para poder generar así profesionales reflexivos que sepan actuar desde la proximidad con los colectivos con los que interactúan pero con un especial sentido de la responsabilidad y del empoderamiento de los usuarios.

La propuesta que se presenta escoge el coaching como herramienta de trabajo ya que es una técnica que acoge a la persona en el presente y la enfoca y la impulsa hacia el futuro, hacia la consecución de sus propósitos. El coaching se evoca para que la persona adquiera su máximo potencial y llegue a la autorrealización, desarrollando a la vez sus competencias intrapersonales e interpersonales. Cabero (2009) habla de coaching emocional, definiéndolo como un proceso dirigido a la consecución de los objetivos emocionales, ya sean dirigidos a uno mismo, a la relación con los demás y al autoliderazgo emocional. Liderar las propias emociones repercute en la persona de manera positiva ya que la protege a la vez que la empodera. Las herramientas propias del coaching permiten incidir en la dimensión emocional de la capacitación profesional. Algunas de estas herramientas son: las preguntas poderosas, la visualización, el rapport, la rueda de la vida, los anclajes, la calibración, la línea del tiempo, el modelo grow, las posiciones perceptivas, las metáforas etc. (Bou, 2007). Es así como el coaching, como proceso formativo e investigador, se observa eficaz, por cuanto contribuye a elevar la conciencia del profesorado con respecto a diversas dimensiones (incluidas las emocionales y motivacionales) que condicionan las formas de actuación en los contextos educativos. En este proceso de formación, el diálogo, es el precursor de los cambios en la conciencia (Jiménez, 2012: 250).

El coaching es una técnica, orientada a resultados de demostrada utilidad en ámbitos educativos y académicos. Sus cinco características esenciales son: la concreción: el coach se enfoca a conductas que pueden ser mejoradas utilizando un lenguaje directo; el diálogo: a través de conversaciones en las que se formulan preguntas y respuestas y se intercambian ideas; la flexibilidad: dado que el coach intenta adaptarse al máximo a las características del coachee, evitando recomendar recetas ajenas a la creatividad y a la voluntad de éste; la responsabilidad compartida: ya que en un clima de respeto mutuo los participantes deben contribuir a que la conversación sea lo más útil posible; y la especificidad de las acciones: atendiendo a objetivos claramente definidos y evaluables (Malagón, 2011). Del mismo modo, Malagón hace una distinción entre coaching educativo y coaching académico, atribuyendo al primero un nivel más general e inclusivo, propio de la educación formal y no formal e informal y al segundo un nivel que puede ser más ajustado a los estudios superiores u otros estudios muy especializados.

El coaching tiene un componente educativo, dado que contribuye de manera eficaz a la consecución de metas personales o grupales y que ello requiere una especial relevancia cuando aplicamos el coaching a la educación de colectivos con dificultades de integración social. Tanto el coaching educativo como el 
académico conducen al alumno a clarificar un problema o dificultad, a cuestionar sus valores, creencias y prácticas concretas, ayudando a identificar los frenos que impiden el desarrollo de las capacidades y a descubrir los recursos en los que puede apoyarse, para crear y aplicar soluciones personales eficaces que le permitan alcanzar sus objetivos. Siguiendo la línea de la importancia del coaching en el trabajo de la competencia emocional, Sánchez y Boronat (2014: 225) lo definen como "un proceso interactivo y sistemático de aprendizaje y de descubrimiento del potencial individual, centrado en el presente y orientado al cambio y la excelencia. Pretende desarrollar la autonomía del alumno, siendo éste el protagonista de los pasos a seguir y de los resultados obtenidos".

El planteamiento curricular que engloba la dimensión emocional, se trabaja de manera interdisciplinar en las asignaturas de carácter procedimental y, a su vez, en el Prácticum, espacio donde en grupos reducidos se profundiza en las características de las figuras profesionales, a la vez que se incide en la dimensión inter e intrapersonal de los estudiantes (Riberas et al., 2014). La tabla 2 muestra las asignaturas que desde ambos Grados trabajan esta dimensión de manera explícita:

Tabla 2: Asignaturas en las que se aborda la competencia emocional

\begin{tabular}{|l|l|l|l|l|}
\hline Asignatura & Créditos & Carácter & Curso & Semestre \\
\hline Habilidades sociales & 6 & Obligatoria & $2^{\circ}$ & $1^{\circ}$ \\
\hline Técnicas de entrevista & 6 & Obligatoria & $2^{\circ}$ & $2^{\circ}$ \\
\hline Gestión de situaciones de crisis & 6 & Obligatoria & $3^{\circ}$ & $1^{\circ}$ \\
\hline Ética profesional & 6 & Obligatoria & $3^{\circ}$ & $1^{\circ}$ \\
\hline Prácticum estratégico de & 33 & Obligatoria & Todos & $1^{\circ}$ y $2^{\circ}$ \\
\hline $\begin{array}{l}\text { Abordaje } \\
\text { situaciones profesionales }\end{array}$ & Optativa & $4^{\circ}$ & $1^{\circ}$ \\
\hline $\begin{array}{l}\text { Pasa ya a la acción, sé tu propio } \\
\text { coach }\end{array}$ & 1,5 & Seminario optativo & Todos & $1^{\circ}$ y $2^{\circ}$ \\
\hline
\end{tabular}

La competencia emocional forma parte del currículum de las dos titulaciones, y concretamente, se trabaja a través de simulaciones y role-playings en las asignaturas de "habilidades sociales" y de "técnicas de entrevista". En estas asignaturas el coaching está presente de manera transversal y forma parte de las herramientas y técnicas que se explican para afrontar la mayoría de los contenidos de las mismas.

La asignatura "Gestión de situaciones de crisis" plantea casos reales donde se debe escoger qué tipo de entrevista y de habilidades sociales y comunicativas habrá que aplicar en función de la edad de la persona entrevistada, el contexto y la gravedad de la situación. De forma simultánea, se analizarán los conflictos de valor propios de esas situaciones críticas, a partir de las habilidades y contenidos que se trabajan en paralelo en la asignatura "Ética profesional". Aprovechando que ese curso realizan prácticas extensivas, los estudiantes realizan un trabajo en esta misma asignatura sobre los conflictos de valor y situaciones críticas en las relaciones inter e intrapersonales que tienen los profesionales de los recursos donde están haciendo estas prácticas, así como las formas de resolverlos. Este tipo de conflictos son ejemplos muy claros de situaciones de complejidad y su análisis permite que el conocimiento de varias disciplinas se integre para dar solución al problema sobre el cual se está trabajando, de tal modo que el aprendizaje no se da en fracciones sino de una manera integral, coherente y dinámica (Fernández y Duarte, 2013: 31). En estas asignaturas, el coaching aporta elementos de reflexión en torno a la gestión de los valores y creencias de las personas, con herramientas que permiten hacer un trabajo, tanto personal como profesional, y donde los estudiantes aprenden a respetar los valores y a trabajar las actitudes y aptitudes para empoderar a los usuarios.

A su vez, el análisis del proceso de prácticas en el espacio de tutoría y supervisión del Prácticum, permite ir ilustrando de forma gráfica, explícita y consciente los contenidos que se están trabajando en las asignaturas. En este espacio se analizan las estrategias que han utilizado los profesionales en la gestión de conflictos y se identifican qué aspectos de las asignaturas de "Habilidades sociales", "Gestión de situaciones de crisis", "Ética profesional" y "Técnicas de entrevista" podrían utilizarse, si tuvieran que hacer un asesoramiento. Además, permite tomar conciencia de las propias capacidades personales y potencialidades, así como de aquellos aspectos que se deberán modificar o reforzar. El coaching por tanto, aporta elementos de reflexión en torno al profesional reflexivo que estamos trabajando y dota a los estudiantes de un espacio de unión entre todas las asignaturas. 
Por otro lado, cabe destacar que toda esta apuesta formativa se complementa con asignaturas optativas como "Abordaje estratégico de situaciones profesionales" donde se refuerza la labor que está realizando el estudiante de cuarto con las prácticas y la supervisión que sigue en el espacio de Prácticum. En esta asignatura se aborda el trabajo sobre estrategias que sumadas al coaching ofrecen un nuevo abanico de recursos para el manejo de situaciones complejas como pueden ser las propias de la Terapia breve estratégica. Además, se ofrece al estudiante la posibilidad de realizar una introducción al coaching con una formación breve en forma de seminario que lleva por nombre "Pasa ya a la acción, sé tu propio coach" donde se muestra el proceso de elaboración de planes de acción para situaciones cotidianas a través de las diferentes técnicas propias del coaching.

A continuación, a modo de ejemplo, se concreta la experiencia realizada en la asignatura de "Técnicas de entrevista". Esta materia, de carácter obligatorio, se imparte en el segundo curso de ambos grados en grupos de 25 alumnos y con una duración de tres horas semanales continuas (donde se da teoría y práctica) durante el segundo semestre del curso. En esta asignatura se incorporan los elementos de la competencia social que se trabajan en el semestre anterior en la asignatura de habilidades sociales, en las diferentes fases de entrevista, mediante la simulación de situaciones profesionales. Se realizan actividades de diversa tipología en las que se pone en juego la autonomía, el autoconocimiento y la autoevaluación por parte de los estudiantes los cuales realizan tareas de mentoring y evaluación entre iguales con el uso de rúbricas que ellos mismos plantean.

Las competencias genéricas de la asignatura son: i) desarrollar habilidades cognitivas, de comunicación y de relación que permitan afrontar de manera adecuada las tensiones y conflictos interpersonales propios del trabajo social y la educación social; ii) comprender los conceptos y ejes fundamentales de la entrevista dentro del proceso metodológico y en el marco disciplinar del trabajo social y la educación social; iii) conocer y lograr técnicas para conducir la entrevista hacia los objetivos profesionales; iv) gestionar el riesgo hacia uno mismo a través de acciones que favorezcan el autoconocimiento, la gestión de las emociones y limiten el estrés profesional; v) poner en práctica los principios y valores del trabajo social y de la educación social, manifestando una actitud respetuosa hacia los destinatarios y hacia los diferentes profesionales, y vi) desarrollar habilidades para documentar procesos de intervención social garantizando la protección de datos y el tratamiento de la información.

Los contenidos se estructuran en cuatro módulos: a) La entrevista en el proceso metodológico de la intervención social; b) El proceso relacional y técnico de la entrevista; c) Habilidades para la conducción de entrevistas, y d) Algunos modelos y contextos de entrevista. Aparte del contenido propio de cada módulo, se quiere destacar aquellas técnicas o herramientas propias del coaching que se han añadido a la asignatura base. A continuación se ejemplifica cada uno de los módulos:

La entrevista en el proceso metodológico de la intervención social. La entrevista es la herramienta por excelencia de todo proceso de acompañamiento socioeducativo (Boadas y Álvarez, 2015). En este módulo se trabaja el contexto, las finalidades, los límites o el papel del profesional. Es aquí donde las herramientas del coaching tienen un papel imprescindible en la autopercepción o la toma de conciencia de los propios procesos cognitivos, emocionales y del nivel competencial en las relaciones intrapersonales e interpersonales. La intervención se basa en el empoderamiento del usuario, dotándole de las herramientas necesarias para la toma de decisiones. El coaching parte de la capacitación y de las potencialidades de la persona. En el aula se ha trabajado esta visión de no protagonismo del profesional y dejar que el usuario sea un agente activo hacia el cambio y la mejora a través de la participación en la formulación de planes de acción. Los ejercicios realizados han sido, entre otros, la elaboración de planes de acción desde esta mirada de protagonismo del usuario, o las simulaciones con usuarios prototípicos que plateaban casos que afectaban al control emocional del profesional (exigiendo la aplicación de técnicas comunicativas; la distancia óptima con la persona atendida; el franqueamiento, o un elevado grado de empatía).

El proceso relacional y técnico de la entrevista. A parte de conocer las fases de la entrevista, el encuadre o el registro de la misma, se ha profundizado en las preguntas poderosas. Estas preguntas son la base del coaching y son aquellas que mueven al cambio y hacen reflexionar al usuario sobre posibles vías de actuación en la resolución de problemas. Se trabaja con los estudiantes la diferente tipología de preguntas: abiertas, cerradas, circulares, preguntas qué, etc. y se practica para adecuar el tipo de entrevista a cada situación a través de casos prácticos o de ejercicios en el aula. La finalidad última es capacitar a los futuros estudiantes en el dominio de formular preguntas para extraer la máxima información, pero también para empoderar a los usuarios. El coaching aquí ha sido clave para reconducir la tendencia de los estudiantes a formular preguntas orientando la respuesta de los usuarios, aportando la idea clave de que las preguntas deben formularse de manera neutra para que sean realmente poderosas. 
Habilidades para la conducción de entrevistas. Este módulo aborda las habilidades básicas y las específicas del proceso de entrevista. La mayoría de ellas coinciden con las herramientas básicas del coaching como pueden ser el rapport, la escucha activa, el parafraseo, etc. y los estudiantes han hecho role-playings guiados por el profesorado para aplicarlas a los casos que se abordan. Este módulo ha sido el básico y más transversal de todos ellos, dado que estas habilidades se han trabajado en cada uno de los ejercicios realizados en el aula. De manera específica, una actividad de grabarse en vídeo haciendo una entrevista desde el rol profesional, ha evidenciado las habilidades de los estudiantes y les ha ayudado a ser conscientes del dominio, o no, de las mismas.

Algunos modelos y contextos de entrevista. El módulo se ha trabajado a través de ponentes expertos, y recoge diferentes contextos de entrevista entre los que se destaca: la entrevista domiciliaria y la entrevista con menores. El coaching se visualizó en la entrevista motivacional, donde desde el pensamiento lateral se crean alternativas en la resolución de problemas (De Bono, 2010). Se enfocó cómo abordar situaciones en las que el usuario es resistente al cambio y cómo a través del diálogo interno puede modificar su visión de la realidad ampliando las alternativas y posibilidades de acción. En consonancia con el resto de módulos, es importante que como futuros profesionales de la acción social los estudiantes sepan dar protagonismo a los usuarios especialmente en las situaciones más críticas. De ahí que se planteara la entrevista motivacional desde el coaching y con la utilización de técnicas creativas para la generación de ideas como la "palabra al azar" que consiste en generar ideas a raíz de vincular la situación con una palabra o, cómo realizar una "lluvia de ideas" correctamente sin juzgar ni priorizar ninguna de las opciones.

A modo general, comentar que se parte de un modelo constructivista que integra los elementos del coaching en el desarrollo de la materia, de manera que el profesor asume el papel de líder-coach y el alumno el de coachee y en algunos casos de coach, cuando se plantean actividades entre iguales, teniendo en cuenta la distinción que hace Peris (2014), en la que establece la diferencia entre coach y líder-coach diferenciándolos en cuanto que el primero se refiere a un mismo posicionamiento - una relación simétrica- y el segundo a cuando se da una situación de poder entre ellos - una relación asimétrica-. El aula se convierte en un espacio de ensayo-error en el que se requiere que los estudiantes tengan una actitud de implicación y de reflexión en el proceso formativo.

Para ello, se trabaja desde la perspectiva de trabajo colaborativo donde se da aprendizaje y evaluación entre iguales y se realizan actividades como el role-playing, el estudio de casos y el modelaje para trabajar en todo momento la conciencia del proceso que cada alumno lleva a cabo, al situarse en una doble dimensión: como observador de sí mismo y como mentor de sus compañeros. La autopercepción sobre las propias competencias, la proyección hacia nuevas estrategias personales y la constante revisión de lo que ocurre en el aula genera procesos que despiertan el interés de los alumnos en la mejora de sus competencias personales y profesionales (Rosa et al., 2014).

\section{RESULTADOS Y DISCUSIÓN}

Una vez concretado el ejemplo de la asignatura de técnicas de entrevista, se presentan los resultados obtenidos en el trabajo sobre la competencia emocional en ambos Grados. Los instrumentos que se han utilizado para evaluar el impacto de esta experiencia formativa son: i) cuestionario de autopercepción de competencias de los estudiantes; ii) cuestionario de evaluación semestral de asignatura; iii) evaluación de los responsables de prácticas sobre las competencias profesionales de los estudiantes, iv) focus grup de estudiantes con evaluadores externos, y v) focus grup de responsables de los centros de prácticas, con evaluador interno (jornada de responsables de prácticas). A continuación se exponen los resultados de cada instrumento:

Cuestionario de autopercepción de competencias de los estudiantes. Para evaluar el nivel de percepción de los estudiantes sobre su grado de adquisición de competencia, se ha diseñado un cuestionario "ad hoc" sobre las competencias emocionales recogidas en las memorias de las titulaciones y se ha pasado al $100 \%$ de la muestra de estudiantes. Se destaca a continuación aquellas que han sido mejor valoradas: i) poner en práctica la escucha activa, la asertividad y el trabajo en equipo $(89,36 \%)$; ii) mostrar una actitud constructiva para resolver cooperativamente los conflictos de valor (83'7\%); iii) identificar las necesidades sociales y reconocer diferentes formas de atenderlas (83'51\%); iv) manifestar conciencia y sensibilidad en relación al compromiso moral que se adquiere en el ejercicio de la profesión ( $\left.83^{\prime} 15 \%\right)$, y v) poner en práctica el sentido crítico y la curiosidad científica como actitudes vinculadas a la profesión (83'15\%).

Cuestionario de evaluación semestral de asignatura. Al finalizar cada semestre, los estudiantes realizan una evaluación anónima de cada una de las asignaturas cursadas. El instrumento recoge elementos de planificación; el desarrollo de las asignaturas; sistema de evaluación; el soporte y la orientación académica, y la actualización e innovación docente del profesorado. En este caso, se percibe una progresión desde 
primer a cuarto curso del $79,15 \%$ al $90,52 \%$ en el Prácticum y de un $76,20 \%$ de satisfacción el primer año de implementación del Grado a un $82,48 \%$ en el $4^{\circ}$ curso, para el conjunto de asignaturas.

Evaluación de los responsables de prácticas sobre las competencias profesionales de los estudiantes. Se trata de una encuesta con preguntas abiertas y cerradas que evalúa las competencias de los estudiantes que han tutorizado en las prácticas. De esta encuesta se recoge que los estudiantes de tercer curso adquieren y manifiestan las competencias previstas en un $86,9 \%$. Este dato se incrementa hasta un $(94,2 \%)$ en los estudiantes de cuarto. En ambos casos, la valoración mediana es del 90,5\%.

Focus grup de estudiantes con evaluadores externos. Los estudiantes valoran de manera muy positiva el trabajo transversal entre asignaturas, especialmente la coherencia en el orden, estructura y secuencia de las mismas. Un aspecto que destaca de manera notable es que adquieren habilidades para afrontar situaciones complejas y estresantes en los entornos donde han realizado las prácticas. Se sienten emocionalmente competentes para dar respuesta a la mayoría de las necesidades detectadas y/o expresadas por los usuarios.

Focus grup de responsables de los centros de prácticas, con evaluador interno (Jornada de responsables de prácticas). Respecto a la valoración del mundo profesional, se realiza una Jornada anual con los responsables de prácticas de los centros con los que se tiene convenio. Además, se realiza una encuesta telemática a los tutores de los centros para recoger su valoración cuantitativa en relación a diversos aspectos del desarrollo de las mismas. El cuestionario consta de preguntas abiertas sobre las competencias de los estudiantes de $3^{\circ}$ y $4^{\circ}$ curso y también recoge propuestas de mejora. Los datos aportan un nivel de adquisición de competencias del $81 \%$ sobre el perfil profesional de los estudiantes.

A partir de estos resultados, y de acuerdo con Sánchez y Boronat (2014), los estudiantes-coachees formados con esta metodología serán capaces de asumir retos y solucionarlos a la vez que crearán otros nuevos que generarán posibilidades e iniciativas que les harán seguir adelante y aprendiendo durante toda su vida. Serán capaces de aplicar esta metodología con los usuarios con los que tengan que trabajar para conseguir con ellos un mejor resultado dado que contarán con una importante variedad de recursos que los llevarán a ser resolutivos y eficaces. Con ello reforzarán a su vez su autoestima y se protegerán minimizando las posibilidades de burnout.

Además, esto implica contribuir a que los niños, adolescentes, jóvenes o adultos con los que trabajen estén en condiciones de superar las dificultades, pero también de aprovecharlas, si se dan las circunstancias, como trampolín de crecimiento y mejora. Esta perspectiva entiende las dificultades y conflictos como parte de la existencia humana, pero, lejos de aceptarlos resignadamente o intentar eludirlos, los afronta abiertamente como oportunidades para desarrollar las posibilidades y potencialidades que estén al alcance de cada persona (López y Rosa, 2014). Por su parte, trabajar desde la Universidad con profesores-coachs incrementa el impacto en el alumnado, por transmitir conocimiento, entusiasmo, respeto y escucha activa (Sánchez y Boronat, 2014). El uso de herramientas del coaching ayuda a que los alumnos expresen sus opiniones y muestren sus emociones desde el respeto, la escucha y la generación de soluciones creativas asumiendo los errores como poderosas herramientas de aprendizaje.

Desarrollar esta práctica educativa partiendo del coaching, aporta resultados positivos desde tres vertientes: metodológica, de prevención de situaciones de burnout y de promotora del cambio. Metodológica, porque trasciende la presentación clásica de los contenidos y se convierte en una estrategia de integración y globalización que integra los conocimientos de diferentes asignaturas, requisito imprescindible para el desarrollo de competencias complejas. De prevención, porque capacita el estudiante, futuro profesional, a gestionar sus emociones y las situaciones adversas con las que, a buen seguro se encontrará. Promotoras de cambio, porque serán estrategias que utilizarán en su actividad profesional para generar procesos de cambio y de transformación en las personas que atenderán.

Así será necesario seguir trabajando para fomentar el desarrollo integral de los futuros profesionales desde una formación de calidad centrada en el crecimiento personal del estudiante, empoderándolo para ser un buen agente del cambio. También, desde una visión crítica con la que pueda poner en práctica todo su potencial para poder trabajar y ofrecer su ayuda a personas en situación de riesgo de exclusión social trabajando la resiliencia y creando planes de acción con los usuarios que les lleve a una mejora de sus condiciones de vida. En esta línea, se está organizando una propuesta de titulación de postgrado: "Experto universitario en coaching de equipos y personas en el ámbito socioeducativo" atendiendo la demanda de estudiantes, de profesorado y de responsables de centros de prácticas. En segundo lugar, se ha publicado el libro titulado "Inteligencia profesional" (Editorial Claret, 2015) que, partiendo del trabajo realizado en las asignaturas de los grados, recoge las principales metodologías que facilitan el desarrollo de la inteligencia emocional. Con todo ello, creemos que se está contribuyendo a crear una mirada que es capaz de 
transcender la perspectiva de las disciplinas clásicas y, de esta manera, responder de manera más eficiente y eficaz a la realidad compleja y siempre cambiante de los entornos profesionales.

La experiencia desarrollada sobre esta forma de acompañamiento presenta también algunas dificultades. Una de ellas es que el propio profesor, y en este caso líder-coach, sea un profesional reflexivo para poder acompañar al estudiante en la revisión de su aprendizaje y de la aplicación de los conocimientos aprendidos en las prácticas. Así mismo, el líder-coach debe ser dominador de sus propias capacidades y competencias emocionales para ejercer de modelo coherente, por lo tanto, es un modelo que incita al profesor a hacer un trabajo personal que lo capacite para ser un buen referente. Una segunda dificultad es la necesaria coordinación permanente y el trabajo cooperativo entre el profesorado de las diferentes asignaturas. Es habitual que el profesorado universitario trabaje de forma poco colaborativa desde su "libertad de cátedra", lo que implica hacer un cambio de posición hacia un trabajo mucho más abierto, compartido y transversal.

También aparece la dificultad de adaptarse a los ritmos del estudiante por parte de profesorado. En estos casos, la aplicación de todo lo expuesto en este artículo quedaría invalidado pues el coaching parte del respeto por los conocimientos y experiencias del otro que le justifican su visión de esa realidad. El acompañamiento por parte del profesor siempre debe mostrar nuevos caminos pero nunca debe forzar, por considerar que son los únicos y mejores, a transitarlos. Esto exige, una revisión de su rol como formador para que realmente se adecue a las exigencias de esta metodología. Desde la visión del estudiante, la propuesta fuerza a los alumnos a ser parte activa y responsable de su proceso de enseñanza-aprendizaje, por lo que alumnos que esperen llegar a clase y ser consumidores pasivos del conocimiento hará que no encuentren motivo ni sentido al trabajo que se realiza.

Finalmente, en el desarrollo de la experiencia se dan diferentes grados de integración de la metodología presentada en función el perfil del docente. En el grupo que el profesorado está capacitado y formado la implicación con la propuesta ha sido mayor, por lo que se ve la necesidad de ofrecer formación en coaching al claustro de las asignaturas que trabajan la dimensión emocional.

Como propuesta de mejora, se ha desplegado una nueva titulación de postgrado que lleva por título "Experto en coaching en el ámbito socioeducativo" que empezará en octubre de 2016 en la que participaran un grupo de profesores motivados para ampliar sus conocimientos y herramientas en esta área. También se seguirá trabajando dentro de la línea de investigación en la que se enmarca esta propuesta para hacer transferencia de conocimiento a otras profesiones; a la supervisión de profesionales en activo; y el acompañamiento y tutorización de los estudiantes en prácticas.

\section{CONCLUSIONES}

De todo lo expuesto se pueden extraer las siguientes conclusiones:

1.- los estudiantes tienen una autopercepción positiva de la formación. Son capaces de establecer relaciones y vínculos entre las distintas asignaturas. Además, toman conciencia de las habilidades, destrezas y conocimientos que han utilizado.

2.- disponen de conocimientos integrados significativamente; tienen un mayor autoconocimiento de sus capacidades, potencialidades y limitaciones, de manera que afrontan con mayores garantías de éxito situaciones imprevistas en los lugares de prácticas.

3.- las experiencias metodológicas de integración entre asignaturas que se desarrollan, contribuyen a la formación de profesionales reflexivos. Les capacita para ejercer la profesión en entornos complejos de manera eficaz, responsable y proactiva ante situaciones potencialmente estresantes.

4.- esta experiencia formativa ha obtenido la acreditación por la auditoría externa que realiza la Agencia de Calidad de Cataluña (AQU), y tiene el reconocimiento de distintos organismos que nos valoran como el mejor centro para cursar estas formaciones.

\section{REFERENCIAS}

Bisquerra, R., Educación emocional y competencias básicas para la vida, Revista de Investigación Educativa, ISSN: 1989-9106 (en línea), 21 (1), 2003. http://revistas.um.es/rie/article/view/99071/94661. Acceso: 2 de octubre (2014) 
Bisquerra, R. y Pérez, N., Las competencias emocionales, Revista Educación XXI, ISSN: 1575-0345 (en línea), $n^{-}$10, 2007. http://revistas.uned.es/index.php/educacionXX1/article/view/297/253. Acceso: 8 de septiembre (2014)

Boadas y Álvarez, La entrevista en contextos de acción social, En Inteligencia profesional, de Riberas y Rosa, pp 97-112, Editorial Claret, Barcelona, España (2015)

Bou Pérez, J.F., Coaching para docentes: el desarrollo de habilidades en el aula, Ecu Narrativa, Alicante, España (2007)

Caride, J.A., Teories, models i paradigmes en Pedagogia-Educació Social, En L'educació Social: projectes, perspectives i camins, pp 47-72, Pleniluni, Barcelona, España (2003)

Cabero, M., El Coaching emocional, Editorial UOC, Barcelona, España (2009)

De Bono, E., El pensamiento creativo. El poder del pensamiento lateral para la creación de nuevas ideas, Paidós Plural, Madrid (2010)

Delors, J., La educación encierra un tesoro. Informe a la UNESCO de la Comisión Internacional sobre la Educación para el siglo XXI, Santillana-Unesco, Madrid 1996. HTTP:/WWW.UNESCO.ORG/EDUCATION/PDF/DELORS_S.PDF. Acceso: 25 de septiembre (2014)

Elliot, J., Pràctica, recerca i teoria en educació, Eumo Editorial, Vic, España (1989)

El Mundo, Las 50 carreras (2014),

http://www.upv.es/entidades/BBAA/menu_urlpi.html?http://www.upv.es/entidades/BBAA/infoweb/fba/info/U0 654837.pdf. Acceso: 27 de abril (2015)

Extremera, N. y Fernández-Berrocal, P., El papel de la inteligencia emocional en el alumnado: evidencias empíricas, Revista Electrónica de Investigación Educativa, ISSN: 1607-4041 (en línea), 6 (2), (2004). http://www.redalyc.org/articulo.oa?id=15506205. Acceso: 3 de octubre (2014)

Fernández, F.H. y Duarte, J.E., El aprendizaje basado en problemas como estrategia para el desarrollo de competencias específicas en estudiantes de ingeniería, Formación universitaria, ISSN: 0718-5006 (en línea), $6 \quad$ (5), 29-38, (2013). http://www.scielo.cl/scielo.php?script=sci_arttext\&pid=S071850062013000500005\&Ing=es\&tIng=es. 10.4067/S0718-50062013000500005. Acceso: 14 de marzo (2015)

Gardner, H., Estructura de la mente: la teoría de las múltiples inteligencias, Fondo de Cultura Económica, México (1987)

Gimeno, J., Teoría de la enseñanza y desarrollo del currículo, Anaya, Madrid, España (1987)

Goleman, D., Inteligencia emocional, Kairós, Barcelona, España (1995)

Imbernon, F., La formación permanente del profesorado, Graó, Barcelona, España (2007)

López, P. y Rosa, G., El desarrollo de competencias para la promoción de la resiliencia: buenas prácticas y procesos formativos en Educación social, Revista Edetania. Estudios y propuestas socioeducativas. ISSN: 0214-8560, N. 45,145-163, (2014)

Madrid Izquierdo, J. M., La formación y la evaluación docente del profesorado universitario ante el espacio europeo de educación superior. Educatio Siglo XXI, (en línea) v. 23, 49-68, (2005). http://revistas.um.es/educatio/article/view/117. Acceso 14 de marzo (2015)

Mayer, J. y Salovey, P., What is emotional intelligence?, En Emotional Development and Emotional Intelligence: Implications for Educators, pp 3-31, Basic Books, Nueva York (1997)

Malagón, F. J., Coaching educativo y académico un nuevo modo de enseñar y aprender, Revista Educación y Futuro, ISSN: 1695-4297 (en línea), 24, (2011). file:///C:/Users/hp/Downloads/DialnetCoachingEducativoYAcademico-3632848.pdf. Acceso: 19 de octubre (2014)

Morin, E., Introducción al pensamiento complejo, Gedisa, Barcelona, España (2008) 
Osuna, C., y Luna, E., Características de ser un Buen Profesional de Ingeniería en la Universidad Autónoma de Baja California, México, Formación universitaria, (en línea), 1(1), 29-36 (2008). http://www.scielo.cl/scielo.php?script=sci_arttext\&pid=S0718-50062008000100005\&lng=es\&tlng=es.

10.4067/S0718-50062008000100005. Acceso: 14 de marzo (2015)

Peris, D., El Coaching como herramienta de transformación para la educación social, Revista Educación social, ISSN: 1698-9097 (en línea), N. 18, (2014). http://www.eduso.net/res/pdf/18/ex28_res_18.pdf. Acceso: 17 de septiembre (2014)

Perrenoud, Ph., Diez nuevas competencias para enseñar, Graó, Barcelona, España (2004)

Ranquing ISSUE de las universidades españolas, Construye tu ranquing de universidades (2015), http://www.u-ranking.es/construye.php. Acceso: 27 de abril (2015)

Riberas, G. y Vilar, J., La praxis reflexiva: un reto para la educación social, Revista Edetania. Estudios y propuestas socioeducativas. ISSN: 0214-8560N, 45, 129-142,(2014)

Riberas, G.; Vilar, J. y Mora, P., Elaboració de projectes socials. Disseny $i$ avaluació de les accions socioeducatives, Claret, Barcelona, España (2013)

Riberas, G.; Vilar, J. y Rosa, G., Nuevas metodologías para la formación de profesionales reflexivos en educación social, Actas del Congreso Internacional de Docencia Universitaria y Innovación, Tarragona, España 2 a 4 de julio (2014)

Rosa, G.; Riberas, G. y Fernández, C., El Prácticum y la capacitación profesional de los estudiantes de Grado de Educación Social y Trabajo Social: de la orientación al desarrollo de competencias, Actas del Congreso Internacional e interuniversitario de orientación educativa y profesional, Málaga, España 18 a 20 de octubre (2012)

Sáez, J., La construcción de la pedagogía social: algunas vías de aproximación, En Pedagogía social, pp 40-67, Ariel, Barcelona, España (1997)

Sánchez, B. y Boronat, J., Coaching Educativo: Modelo para el desarrollo de competencias intra e interpersonales. Revista Educación XXI, ISSN: 1575-0345 (en línea), 17 (1), (2014). file:///C:/Users/hp/Downloads/10712-17561-1-PB.pdf. Acceso: 8 de octubre (2014)

Salvador, L.; Argos, J.; Ezquerra, M.P.; Osoro, J.M.I y Castro, A., Perfiles de estilos de aprendizaje de los estudiantes universitarios y metodologías docentes. Bordón, ISSN: 0210-5934 -41 (en línea), 63 (2), (2011). http://recyt.fecyt.es/index.php/BORDON/article/view/28970. Acceso: 6 de mayo (2015)

Schön, D., El profesional reflexivo. Cómo piensan los profesionales cuando actúan, Paidós, Barcelona, España (1998)

Secretaría de Estado de Universidades e Investigación, Propuestas para la renovación de las metodologías educativas en la universidad, Madrid: Ministerio de educación y ciencia. (2006).http://www.catedraunesco.es/archivos/metodologias.pdf. Acceso: 8 octubre (2014)

Stenhouse, L., La investigación como base de la enseñanza, Morata, Madrid, España (1987)

Tobón, S., Formación basada en competencias, Ecoe, Bogotá (2005)

Trillo, F., El Espacio Europeo de Educación Superior (EEES): planteando preguntas para establecer coordenadas, Educación social. Revista de intervención socioeducativa, N. 40, 13-29 (2008)

Úcar, X., El porqué y el para qué de la pedagogía social, En La pedagogía social en la sociedad de la información, 233-270, Editorial UOC, Barcelona, España (2006)

Viñas, A. y Fabregó, E., El professional com a persona, Revista Papers, ํㅡ 24, Barcelona: Departament de Benestar Social i Família. Generalitat de Catalunya, (2012). http://www20.gencat.cat/docs/bst/01Departament/08Publicacions/Coleccions/Papers\%20acci\%C3\%B3\%20s ocial/num_24/papers_24.pdf. Acceso: 3 de octubre (2014) 
